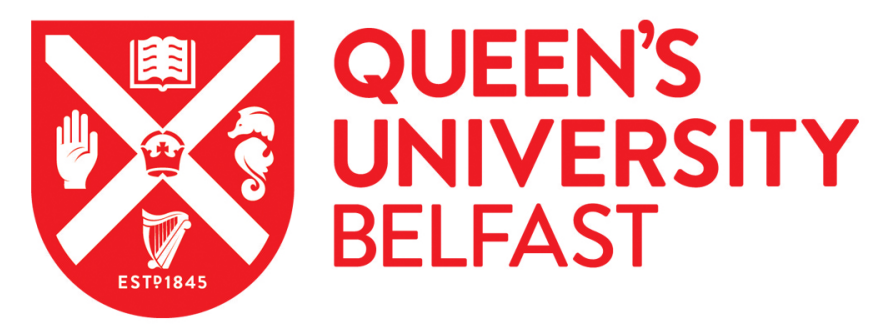

\title{
High-order harmonic generation from highly excited states in acetylene
}

Mulholland, P., \& Dundas, D. (2018). High-order harmonic generation from highly excited states in acetylene. Physical Review A (Atomic, Molecular, and Optical Physics), 97, [043428].

https://doi.org/10.1103/PhysRevA.97.043428

Published in:

Physical Review A (Atomic, Molecular, and Optical Physics)

Document Version:

Publisher's PDF, also known as Version of record

Queen's University Belfast - Research Portal:

Link to publication record in Queen's University Belfast Research Portal

Publisher rights

@2018 American Physical Society. This work is made available online in accordance with the publisher's policies. Please refer to any applicable terms of use of the publisher.

\section{General rights}

Copyright for the publications made accessible via the Queen's University Belfast Research Portal is retained by the author(s) and / or other copyright owners and it is a condition of accessing these publications that users recognise and abide by the legal requirements associated with these rights.

Take down policy

The Research Portal is Queen's institutional repository that provides access to Queen's research output. Every effort has been made to ensure that content in the Research Portal does not infringe any person's rights, or applicable UK laws. If you discover content in the Research Portal that you believe breaches copyright or violates any law, please contact openaccess@qub.ac.uk. 


\title{
High-order harmonic generation from highly excited states in acetylene
}

\author{
Peter Mulholland and Daniel Dundas \\ Atomistic Simulation Centre, School of Mathematics and Physics, Queen's University Belfast, University Road, \\ Belfast BT7 1NN, Northern Ireland, United Kingdom
}

(Received 10 March 2017; revised manuscript received 30 October 2017; published 25 April 2018)

\begin{abstract}
High-order harmonic generation (HHG) from aligned acetylene molecules interacting with mid infra-red (IR), linearly polarized laser pulses is studied theoretically using a mixed quantum-classical approach in which the electrons are described using time-dependent density-functional theory while the ions are treated classically. We find that for molecules aligned perpendicular to the laser polarization axis, HHG arises from the highest-occupied molecular orbital (HOMO), while for molecules aligned along the laser polarization axis, HHG is dominated by the HOMO-1. In the parallel orientation we observe a double plateau with an inner plateau that is produced by ionization from and recombination back to an autoionizing state. Two pieces of evidence support this idea. First, by choosing a suitably tuned vacuum ultraviolet pump pulse that directly excites the autoionizing state we observe a dramatic enhancement of all harmonics in the inner plateau. Second, in certain circumstances, the position of the inner plateau cutoff does not agree with the classical three-step model. We show that this discrepancy can be understood in terms of a minimum in the dipole recombination matrix element from the continuum to the autoionizing state.
\end{abstract}

DOI: 10.1103/PhysRevA.97.043428

\section{INTRODUCTION}

High-order harmonic generation (HHG) is a highly nonlinear process in which an atom or molecule absorbs energy from an intense laser pulse before emitting short attosecond bursts of radiation with a frequency that can be many multiples of the incident laser frequency. Understanding and controlling HHG is crucial since it provides a versatile tool for a range of different applications including the use of high harmonic spectroscopy to probe chemical reactions [1], using it as a tool for imaging individual molecular orbitals [2] and for producing attosecond pulse trains [3]. High-order harmonic generation is usually described using the classical three-step model in which an electron is ionized by the laser pulse and subsequently driven far from the parent ion by the field before finally recombining with the parent with the resultant emission of radiation $[4,5]$.

Generally, it is assumed that the electron ionizes from and returns to the ground state. However, many studies now propose different schemes involving excitation to intermediate states that can greatly influence the generation of harmonics. For example, early studies of HHG from a coherent superposition of states showed that multiple plateaus can be produced with cutoffs associated with the ionization potential of the states involved [6]. Four-step models of HHG have also been developed to describe how autoionizing states can influence the harmonic response [7]. In addition, studies of HHG in asymmetric diatomic molecules have observed double plateau structures which have been ascribed to resonant excitation induced by laser-induced electron transfer [8]. In that case, HHG is associated with a channel in which electrons are ionized from an excited state and recombine to the ground state. Recently, this channel was observed experimentally in studies of HHG in argon atoms [9]. In many cases the enhancement is only observed over a narrow band of harmonics $[10,11]$.
However, by exciting atomic targets with an IR pulse in conjunction with a high-order harmonic pulse, a dramatic enhancement of the harmonic spectra across a wide frequency range was observed $[12,13]$.

In this work we show that highly excited states play an important role in HHG from aligned acetylene molecules interacting with mid infra-red (IR) linearly polarized laser pulses $(\lambda=1450 \mathrm{~nm})$. Using such long laser wavelengths allows the production of an extended plateau using laser intensities well below the saturation limit [1]. Indeed, several studies of HHG in acetylene have already been carried out [14-17]. These have mainly concentrated on studying the role of structural interference minima [18,19]. Acetylene is a small, linear polyatomic molecule with the ground-state configuration $\left(1 \sigma_{g}\right)^{2}\left(1 \sigma_{u}\right)^{2}\left(2 \sigma_{g}\right)^{2}\left(2 \sigma_{u}\right)^{2}\left(3 \sigma_{g}\right)^{2}\left(1 \pi_{u}\right)^{4}$. In addition, the next lowest unoccupied orbitals are $\left(1 \pi_{g}\right)^{0}\left(3 \sigma_{u}\right)^{0}\left(4 \sigma_{g}\right)^{0}$. It is widely known that resonance phenomena greatly influence photoabsorption and photoionization cross sections in acetylene [2028]. While many studies have considered excitations from the highest occupied molecular orbital (HOMO) to the lowest unoccupied molecular orbital (LUMO), there is much interest in the role of highly excited states in photoabsorption and photoionization at vacuum ultraviolet (VUV) wavelengths [2328]. Therefore, we can envisage that these highly excited states can give rise to several possible mechanisms for HHG.

The paper is arranged as follows. In Sec. II we describe our approach for describing HHG in acetylene, namely, timedependent density-functional theory (TDDFT). In particular, we detail the various numerical parameters used in the simulations reported here. Section III then presents our results. First, we show how our calculations give an accurate description of the static properties of acetylene, in terms of ionic configurations, ionization potentials, excited states, etc. Then we present calculations of HHG in acetylene using a 
range of short-duration laser pulses with wavelengths ranging from VUV to mid-IR wavelengths. These simulations show that populating particular highly excited states using a VUV pulse greatly enhances the harmonic yield produced through interaction with a mid-IR pulse. In addition, we present the populations in a range of states in order to provide information on those states that are excited during interaction with the laser pulses. Finally, in Sec. IV some conclusions are drawn.

Unless otherwise stated, atomic units are used throughout.

\section{METHOD}

Our calculations are carried out using TDDFT [29], as implemented in our code EDAMAME [30,31]. EDAMAME is a highly parallelized implementation of the nonadiabatic quantum molecular dynamics method, in which the electronic dynamics are calculated quantum mechanically using TDDFT on a real-space grid, while the ionic motion is treated classically. While TDDFT has well-known problems in describing autoionizing resonances arising from double excitations, it does capture those arising from single excitations [32]. For the resonance phenomena studied in this work, previous TDDFT studies of photoionization of acetylene have shown good accuracy [28].

The electronic dynamics are modeled by solving the timedependent Kohn-Sham (TDKS) equations

$$
i \frac{\partial}{\partial t} \psi_{j}(\boldsymbol{r}, t)=H_{\mathrm{KS}} \psi_{j}(\boldsymbol{r}, t), \quad j=1, \ldots, N,
$$

with the Kohn-Sham Hamiltonian $H_{\mathrm{KS}}$ defined as

$$
H_{\mathrm{KS}}=-\frac{1}{2} \nabla^{2}+V_{\mathrm{H}}(\boldsymbol{r}, t)+V_{\mathrm{ext}}(\boldsymbol{r}, \boldsymbol{R}, t)+V_{\mathrm{xc}}(\boldsymbol{r}, t),
$$

where $V_{\mathrm{H}}(\boldsymbol{r}, t)$ is the Hartree potential, $V_{\mathrm{ext}}(\boldsymbol{r}, \boldsymbol{R}, t)$ is the external potential, $V_{\mathrm{xc}}(\boldsymbol{r}, t)$ is the exchange-correlation potential, and $\psi_{j}(\boldsymbol{r}, t)$ are the Kohn-Sham orbitals. Neglecting spin effects, the time-dependent electron density is then calculated as

$$
n(\boldsymbol{r}, t)=2 \sum_{j=1}^{N}\left|\psi_{j}(\boldsymbol{r}, t)\right|^{2}
$$

where each Kohn-Sham orbital has an initial occupation of 2, and $N=N_{e} / 2$, where $N_{e}$ is the number of active electrons (for clarity here we have neglected spin degrees of freedom).

The exchange-correlation potential $V_{\mathrm{xc}}(\boldsymbol{r}, t)$ in Eq. (2), which accounts for all electron-electron interactions in the TDKS equations, is approximated using the local-density approximation incorporating the Perdew-Wang parametrization of the correlation functional [33]. This functional contains self-interaction errors, which means that its long-range behavior is incorrect. One major consequence is that electrons are too loosely bound and excited states are not accurately described [34]. Therefore, we supplement this functional with the average density self-interaction correction (ADSIC) [35], which reinstates the correct long-range behavior in an orbitalindependent fashion.

The Kohn-Sham orbitals in EDAMAME are discretized on three-dimensional finite-difference grids in Cartesian coordinates. The grid spacing was taken to be $0.4 a_{0}$ in each direction and the grid extents were $|x|=|y| \leqslant 90.8 a_{0}$ and $|z| \leqslant 146.8 a_{0}$. Absorbing boundaries are implemented near the edges of the grid using a mask function technique [30] in order to prevent wave-packet reflections from the edges of the grid.

The external potential $V_{\text {ext }}(\boldsymbol{r}, \boldsymbol{R}, t)$ accounts for both the interaction of the laser field with the electrons and the electronion interactions. The laser-interaction term is described within the dipole approximation in the length gauge. The electric field vector $\boldsymbol{E}(t)$ is defined through its relationship to the vector potential $\boldsymbol{A}(t)$, namely,

$$
\boldsymbol{E}(t)=-\frac{\partial}{\partial t} \boldsymbol{A}(t)
$$

All calculations presented in this paper consider acetylene interacting with linearly polarized pulses described by $\sin ^{2}$ pulse envelopes [30]

$$
f(t)= \begin{cases}\sin ^{2}\left(\frac{\pi t}{T}\right) & 0 \leqslant t \leqslant T \\ 0 & \text { otherwise }\end{cases}
$$

where $T$ is the pulse duration. In this case the vector potential is defined as

$$
\boldsymbol{A}(t)=A_{0} f(t) \cos \left(\omega_{L} t\right) \hat{\boldsymbol{e}},
$$

from which $\boldsymbol{E}(t)$ can be written as

$$
\boldsymbol{E}(t)=\left(E_{0} f(t) \sin \left(\omega_{L} t\right)-\frac{E_{0}}{\omega_{L}} \frac{\partial f}{\partial t} \cos \left(\omega_{L} t\right)\right) \hat{\boldsymbol{e}} .
$$

Here $A_{0}$ and $E_{0}$ are the peak values of the vector potential and electric field vector, respectively, $\omega_{L}$ is the laser frequency, and $\hat{\boldsymbol{e}}$ is the unit vector in the polarization direction of the laser field.

For the pulses considered, we assume that the innermost electrons do not contribute to the dynamical response and therefore only consider the response of the ten outermost electrons. The electron-ion interactions are described with Troullier-Martins pseudopotentials [36] in the KleinmanBylander form [37]. All pseudopotentials were generated using the Atomic Pseudopotential Engine (APE) [38].

The ionic motion is treated classically using Newton's equations of motion. For ion $k$ we have

$$
\begin{aligned}
M_{k} \ddot{\boldsymbol{R}}_{k}= & -\int n(\boldsymbol{r}, t) \frac{\partial H_{\mathrm{KS}}}{\partial \boldsymbol{R}_{k}} d \boldsymbol{r} \\
& -\frac{\partial}{\partial \boldsymbol{R}_{k}}\left[V_{n n}(\boldsymbol{R})+Z_{k} \boldsymbol{R}_{k} \cdot \boldsymbol{E}(t)\right],
\end{aligned}
$$

where $V_{n n}(\boldsymbol{R})$ is the Coulomb repulsion between the ions and $Z_{k} \boldsymbol{R}_{k} \cdot \boldsymbol{E}(t)$ denotes the interaction between ion $k$ and the laser field.

The TDKS equations (1) are propagated in time using an 18th-order Arnoldi propagator, while the ionic equations of motion (8) are propagated in time using the velocity Verlet method. The time step in both cases was 0.2 a.u.

\section{RESULTS}

In this section we present results for the interaction of acetylene with intense laser pulses. In particular we consider the harmonic response of the molecule while at the same time considering the excitation and ionization dynamics. First, 
we describe how these quantities are calculated using our approach.

We calculate HHG spectra by taking the Fourier transform of the dipole acceleration, along the direction $\hat{\boldsymbol{e}}_{k}$ [39],

$$
S_{k}(\omega)=\left|\int e^{i \omega t} \hat{\boldsymbol{e}}_{k} \cdot \ddot{\boldsymbol{d}}(t) d t\right|^{2} .
$$

Here $\ddot{\boldsymbol{d}}(t)$ is the dipole acceleration, which is given by

$$
\ddot{\boldsymbol{d}}(t)=-\int n(\boldsymbol{r}, t) \nabla H_{\mathrm{KS}} d \boldsymbol{r} .
$$

Note that all the figures in this work present the spectral density along the laser polarization direction; $S_{k}(\omega)$ is negligible along the other directions.

Additional information can be gained by calculating the response for each Kohn-Sham orbital [40]. In that case we calculate the dipole acceleration from each Kohn-Sham state as

$$
\begin{aligned}
\ddot{\boldsymbol{d}}_{j}(t) & =-\int n_{j}(\boldsymbol{r}, t) \nabla H_{\mathrm{KS}} d \boldsymbol{r} \\
& =-2 \int\left|\psi_{j}(\boldsymbol{r}, t)\right|^{2} \nabla H_{\mathrm{KS}} d \boldsymbol{r}, \quad j=1, \ldots, N .
\end{aligned}
$$

While this neglects interferences between different orbitals in the overall harmonic signal, it does give an indication of the contribution of that state. To gain insight into the excitation processes occurring during (and following) the VUV pulse, we have calculated the overlap of the $N$ time-dependent KohnSham orbitals $\psi_{j}(\boldsymbol{r}, t)$ with the lowest-energy occupied and unoccupied field-free Kohn-Sham orbitals, namely,

$$
\eta_{k}(t)=2 \sum_{j=1}^{N}\left|\int \phi_{k}^{*}(\boldsymbol{r}) \psi_{j}(\boldsymbol{r}, t) d \boldsymbol{r}\right|^{2}, \quad k=1, \ldots, M,
$$

where $\phi_{k}(\boldsymbol{r})$ are the $M(M>N)$ lowest-energy field-free eigenstates of the Kohn-Sham Hamiltonian.

\section{A. Static properties}

Using the pseudopotentials and exchange-correlation functional detailed above, the $X^{1} \Sigma_{g}{ }^{+}$ground state of the molecule was calculated. This gave a C-C bond length of $2.207 a_{0}$ while the $\mathrm{C}-\mathrm{H}$ bond length was $2.045 a_{0}$. These agree well with the experimental values of $2.273 a_{0}$ and $2.003 a_{0}$, respectively [27]. For this equilibrium geometry, both occupied and unoccupied Kohn-Sham orbitals were calculated. The energies of these orbitals are shown in Fig. 1. By Koopmans' theorem, the magnitude of the HOMO energy can be considered as a good approximation to the vertical ionization potential to the $X^{2} \Pi_{u}$ cationic state. Similarly, the magnitudes of the HOMO-1 and HOMO-2 energies can be thought of as approximations to the energies required to ionize to the $A^{2} \Sigma_{g}{ }^{+}$and $B^{2} \Sigma_{u}{ }^{+}$cationic states, respectively. For these three cationic states, the experimental ionization potentials are $0.4191,0.6140$, and 0.6912 hartree, respectively [41]. Our calculated values for these states are $0.4149,0.5548$, and 0.6117 hartree, respectively.

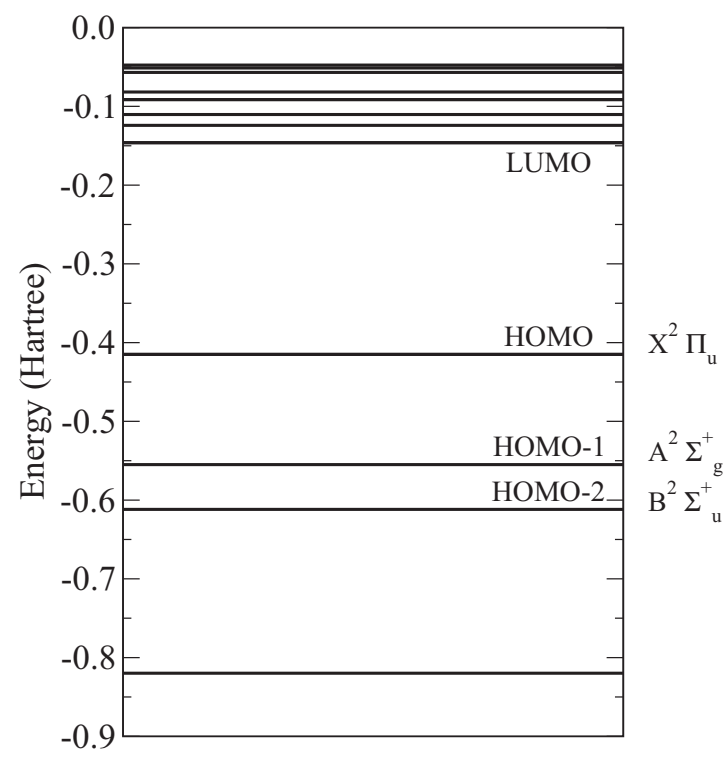

FIG. 1. Occupied and unoccupied Kohn-Sham orbital energies of acetylene, obtained from a geometry-relaxed calculation of the ground state using the ADSIC exchange-correlation functional. From Koopmans' theorem, the magnitude of the HOMO, HOMO-1, and HOMO-2 energies approximate ionization potentials to the ground state $\left(X^{2} \Pi_{u}, 0.4191\right.$ hartree $)$, first excited state $\left(A^{2} \Sigma_{g}{ }^{+}, 0.6140\right.$ hartree), and second excited state $\left(B^{2} \Sigma_{u}{ }^{+}, 0.6912\right.$ hartree) of the acetylene cation, respectively.

\section{B. High-order harmonic generation: The IR pulse}

Consider the interaction of acetylene with a five-cycle (24.2-fs) laser pulse having a wavelength of $\lambda=1450 \mathrm{~nm}$ (photon energy equal to 0.0314 hartree) and a peak intensity of $I=1.0 \times 10^{14} \mathrm{~W} / \mathrm{cm}^{2}$. Figure 2 presents harmonic spectra for acetylene aligned both parallel and perpendicular to the laser polarization direction. We see that the intensity of the plateau harmonics is several orders of magnitude larger when the molecule is aligned perpendicular to the laser polarization direction; this well-known result is based on the symmetry of the HOMO. However, two additional features are present in the plots. First, for the parallel orientation, we see evidence of a double plateau with an inner plateau cutoff near harmonic 65. Second, the position of the outer cutoff is different for each orientation. For the perpendicular orientation, the position of the cutoff is consistent with ionization from the HOMO, whereas for the parallel orientation the cutoff is consistent with ionization from the HOMO-1. We note that the parallel cutoff is also consistent with ionization from the HOMO-2. We have calculated the HHG spectra arising from each occupied KohnSham orbital [40]; these are shown in Figs. 3(a) and 3(b) for the parallel and perpendicular orientations, respectively. These results suggest that in the perpendicular orientation HHG arises from the HOMO while in the parallel case HHG arises predominantly from the HOMO-1, especially in the cutoff region. Extensions to the cutoff due to ionization from different orbitals have already been observed in other molecules such as $\mathrm{N}_{2}$ [42].

Previous studies of photoabsorption and photoionization in acetylene have considered the role of highly excited states 


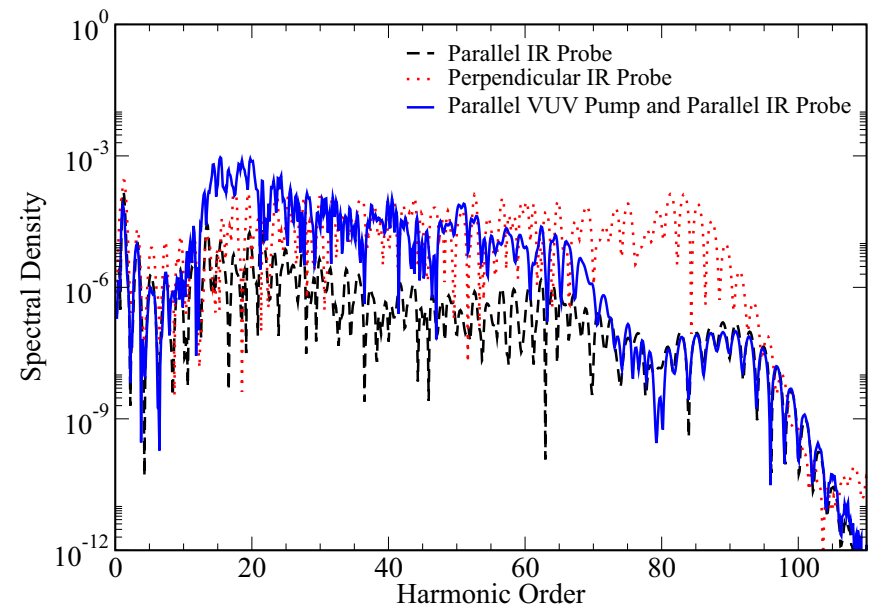

FIG. 2. High-order harmonic generation in acetylene after interaction with a five-cycle, linearly polarized IR laser pulse having a wavelength of $\lambda=1450 \mathrm{~nm}$ and a peak intensity of $I=$ $1.0 \times 10^{14} \mathrm{~W} / \mathrm{cm}^{2}$. Two orientations of the molecule with the field were considered, namely, the parallel and perpendicular orientations. Additionally, we plot the harmonic spectra for the parallel orientation in which the IR pulse has been immediately preceded by an eightcycle, linearly polarized VUV laser pulse having a wavelength of $\lambda=102 \mathrm{~nm}$ and a peak intensity of $I=1.0 \times 10^{12} \mathrm{~W} / \mathrm{cm}^{2}$.

[23-28]. In particular, features in the photoionization spectrum around 0.4963 hartree in the photon energy are generally associated with the formation and subsequent autoionization of the $3 \sigma_{g} \rightarrow 3 \sigma_{u}$ excited state [26,27]. Such a transition is associated with an excitation from the HOMO-1 to the LUMO+1. In that case, for HHG in the parallel orientation we could envisage a situation in which an electron is excited to the LUMO+1, ionized from this state, and then recombined back to it. In our simulations, the LUMO+1 energy is -0.1103 hartree and so HHG from the LUMO+1 would give a cutoff at

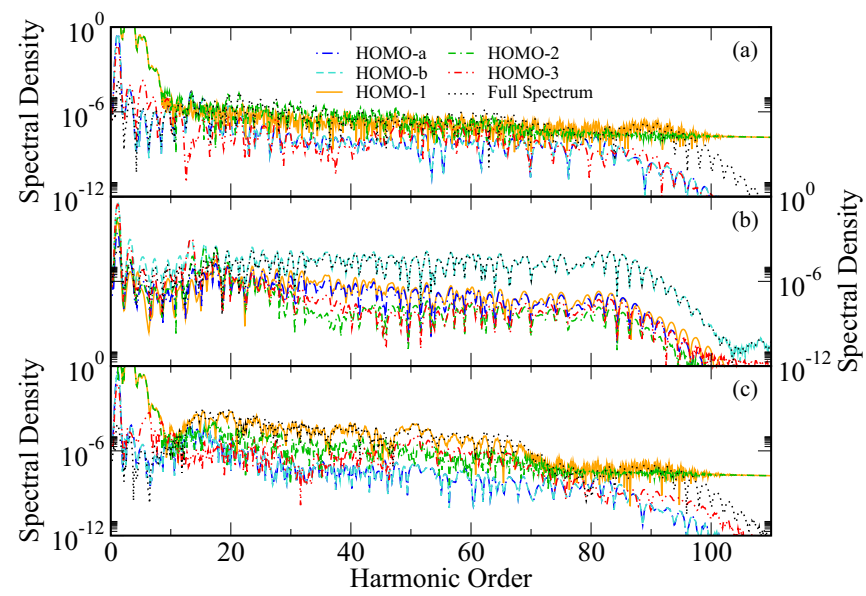

FIG. 3. Contributions of individual Kohn-Sham orbitals to the three harmonic spectra shown in Fig. 2, calculated using Eq. (11), along with the full harmonic signals for reference: (a) parallel and (b) perpendicular orientations with only the IR probe present and (c) spectra for the parallel-pump-parallel-probe case described in the text. In these plots, HOMO-a and HOMO-b refer to the two forms of the HOMO.

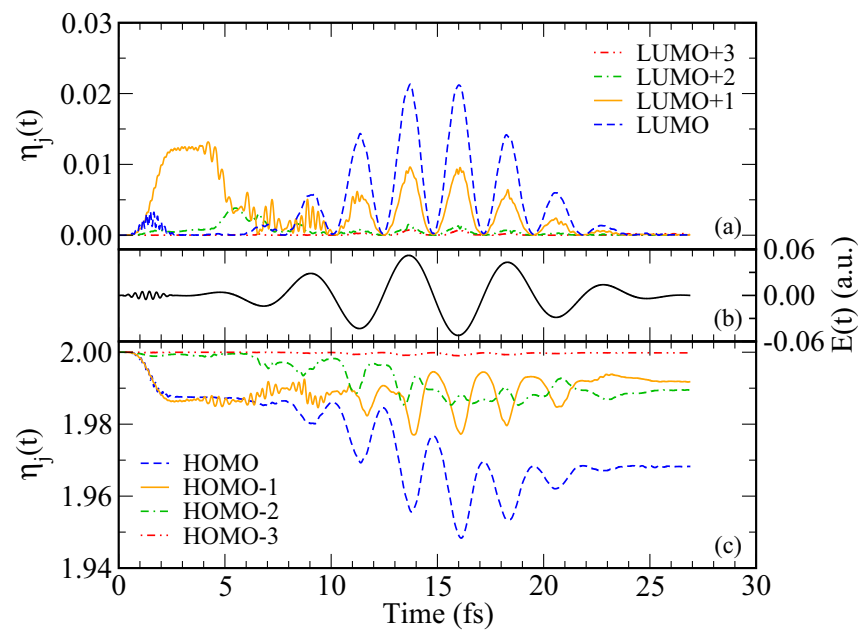

FIG. 4. Electronic population in each of the ten lowest initial Kohn-Sham orbitals $\phi_{k}(\boldsymbol{r})$, calculated using Eq. (12) during interaction with an eight-cycle, 102-nm VUV pump pulse with peak intensity $1.0 \times 10^{12} \mathrm{~W} / \mathrm{cm}^{2}$ followed by a five-cycle, $1450-\mathrm{nm}$ IR probe with peak intensity $1.0 \times 10^{14} \mathrm{~W} / \mathrm{cm}^{2}$ for the (a) lowest unoccupied and (c) highest occupied orbitals. (b) The $z$ component of the electric field vector $\boldsymbol{E}(t)$ is provided for reference. For brevity, only one form of each of the HOMOs and LUMOs is shown.

harmonic 77. This does not agree with the observed cutoff for the inner plateau around harmonic 65 . We will return to this point later.

\section{High-order harmonic generation: The VUV pulse plus the IR pulse}

In order to investigate the role of the $3 \sigma_{g} \rightarrow 3 \sigma_{u}$ excitation, we can excite the molecule using an eight-cycle (2.67-fs) linearly polarized VUV laser pulse having a wavelength of $\lambda=102 \mathrm{~nm}$ (photon energy equal to 0.4467 hartree) and a peak intensity of $I=1.0 \times 10^{12} \mathrm{~W} / \mathrm{cm}^{2}$. For this pulse, the bandwidth is sufficient to also excite the $2 \sigma_{u} \rightarrow 4 \sigma_{g}$ transition. Immediately after the pump pulse, the molecule interacts with the IR laser pulse. The polarization direction of both pulses is along the molecular axis. The resulting harmonic spectra are shown in Fig. 2. Two areas of significant harmonic enhancement are observed. First, a window of enhanced harmonics (harmonics 11-21) is present. Second, the inner secondary plateau that was observed for the IR-only pulse is greatly enhanced. Indeed, the intensity of the harmonics in the inner plateau is now comparable to that using the IR probe aligned perpendicular to the molecular axis. This enhancement could arise from either the $3 \sigma_{g} \rightarrow 3 \sigma_{u}$ or $2 \sigma_{u} \rightarrow 4 \sigma_{g}$ excitations. If we calculate the $\mathrm{HHG}$ spectra arising from each occupied Kohn-Sham orbital [Fig. 3(c)] we see that this plateau arises from the HOMO-1. Furthermore, consider Fig. 4, which shows the calculated overlaps of the time-evolving Kohn-Sham orbitals with the initial orbitals. Of particular interest are the overlaps with the LUMO+1 and LUMO+2. We clearly see during the interaction that it is the LUMO+1 that is excited. Hence we attribute the enhancement in the HHG spectra to the $3 \sigma_{g} \rightarrow 3 \sigma_{u}$ excitation.

Returning now to the pump-probe spectrum shown in Fig. 2, we can identify two mechanisms that give rise to the observed 

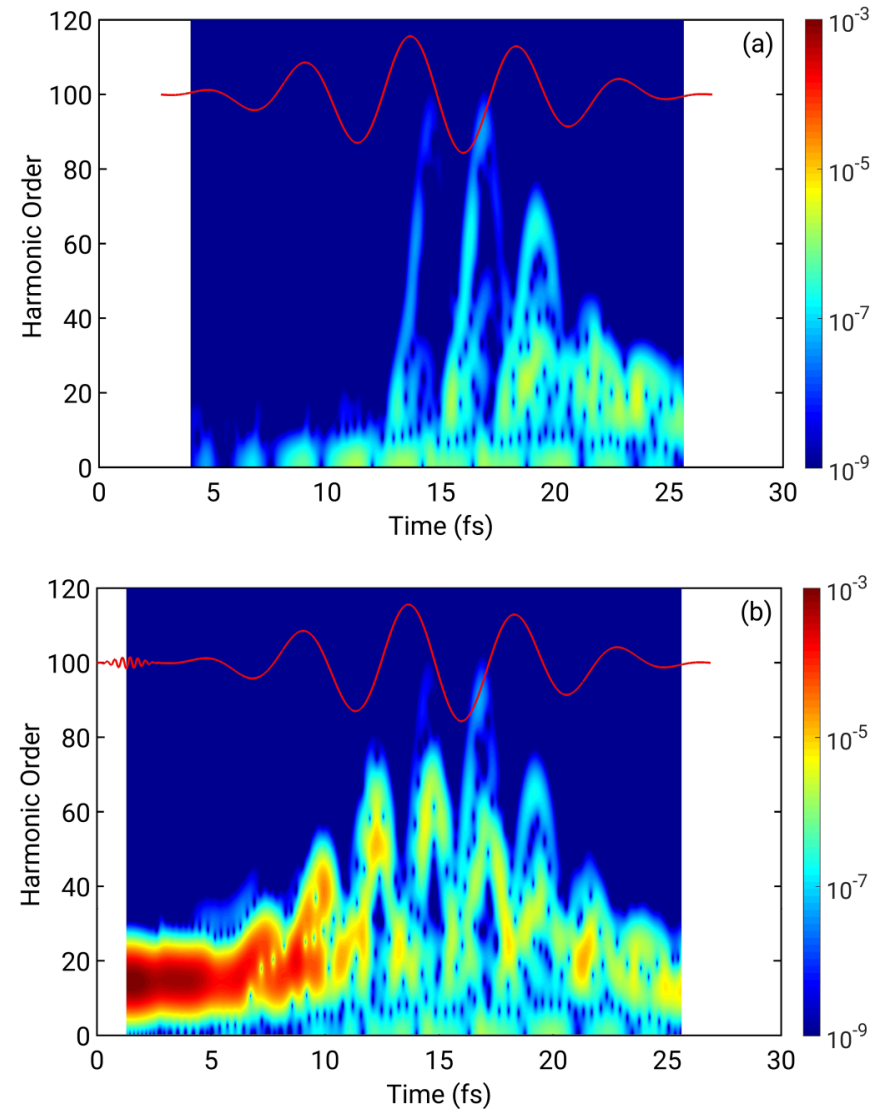

FIG. 5. Time frequency analysis of the two HHG spectra obtained in the parallel orientation in Fig. 2. Results were obtained using (a) the IR pulse only and (b) a VUV pump pulse immediately preceding the IR pulse. The red lines at the top of each plot denote the electric field.

harmonic enhancements. After excitation of the molecule by the VUV pump pulse, relaxation can now occur back to the ground state, resulting in the emission of a high-energy photon. For a VUV photon energy of 0.4467 hartree, this would correspond to harmonic 15 of the IR pulse. The enhancement for harmonics $11-21$ is thus due to bound-bound transitions as the molecule relaxes back to the ground state. For the inner plateau, the enhancement appears to originate due to ionization from and subsequent recombination back to the excited state. This explanation is backed up by carrying out a time-frequency analysis of the harmonic response. Figure 5(a) presents the time-frequency spectrum for the IR-only pulse in the parallel orientation while Fig. 5(b) presents the results for the VUV plus IR pulses. For the IR-only results we see that the plateau harmonics are not emitted until the pulse has ramped on fully. This is consistent with the normal three-step model. During the initial ramp-on, excitation also occurs which can lead to subsequent HHG from the excited state. For the VUV plus IR pulses the situation is dramatically different. In this case we see the low-order harmonic enhancement occurring during the interaction with the VUV pump right up until the first few cycles of the IR pulse. At this point these bound-bound transitions cease as the excited-state population ionizes before recombining back to the excited state. These bound-continuum transitions occur much earlier during the IR pulse as the

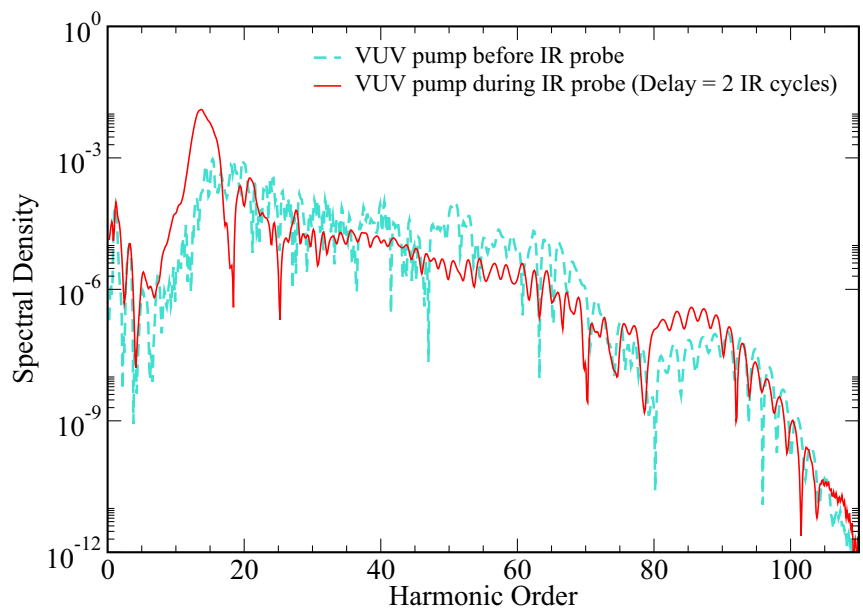

FIG. 6. Harmonic spectra from acetylene with different setups of pump and probe pulses. The VUV pump pulse is an eightcycle pulse with wavelength $\lambda=102 \mathrm{~nm}$ and a peak intensity of $I=1.0 \times 10^{12} \mathrm{~W} / \mathrm{cm}^{2}$, while the IR probe pulse is a five-cycle pulse with wavelength $\lambda=1450 \mathrm{~nm}$ and a peak intensity of $I=$ $1.0 \times 10^{14} \mathrm{~W} / \mathrm{cm}^{2}$. All of the pulses are linearly polarized along the molecular axis.

population is already excited. Thus we see the formation of the plateau well before the pulse has ramped up to maximum intensity. As the IR pulse ramps off, HHG switches off and population in the excited state now deexcites to the ground state, resulting in the generation of low-order harmonics again.

We have also investigated the effect of changing the delay between the VUV pump and IR probe pulses. The harmonic spectrum resulting from one such calculation is shown in Fig. 6. In this case the VUV pulse is applied during the IR pulse, beginning two cycles into the IR pulse (in the following, we call this the overlapping pulses setup). We observe less enhancement of the plateau harmonics compared with the case where the VUV pulse precedes the IR pulse (we call this the sequential pulses setup). This is because a VUV pulse applied to the ground-state molecule before the IR pulse is much more effective at exciting the $3 \sigma_{g} \rightarrow 3 \sigma_{u}$ transition than one applied during the IR pulse, when the system has already been excited. The window of harmonics due to bound-bound transitions also differs for the two setups as shown in Fig. 6. In the sequential case, the VUV pulse is applied when the molecule is in its ground state. During the interaction with this pump pulse, the LUMO+1 is populated, with the subsequent transitions back to the ground state producing relatively-well-defined harmonics. For the overlapping pulses, the situation is different. Now the VUV pulse is being applied when the system is already far from equilibrium and where some population may have been excited to the LUMO+1 by the IR pulse. Applying the VUV pump in this case can excite more transitions to the LUMO+1, as before, but can also stimulate the emission of the low-order (bound-bound) harmonics. The broadening of the peak reflects the distorted nature of the molecule at the time when the VUV pulse is applied.

Rather than overlapping the two pulses, we could instead increase the delay time between them. As might be expected, the spectra produced in these cases (not shown here) are similar to the spectrum produced for sequential pump and probe 


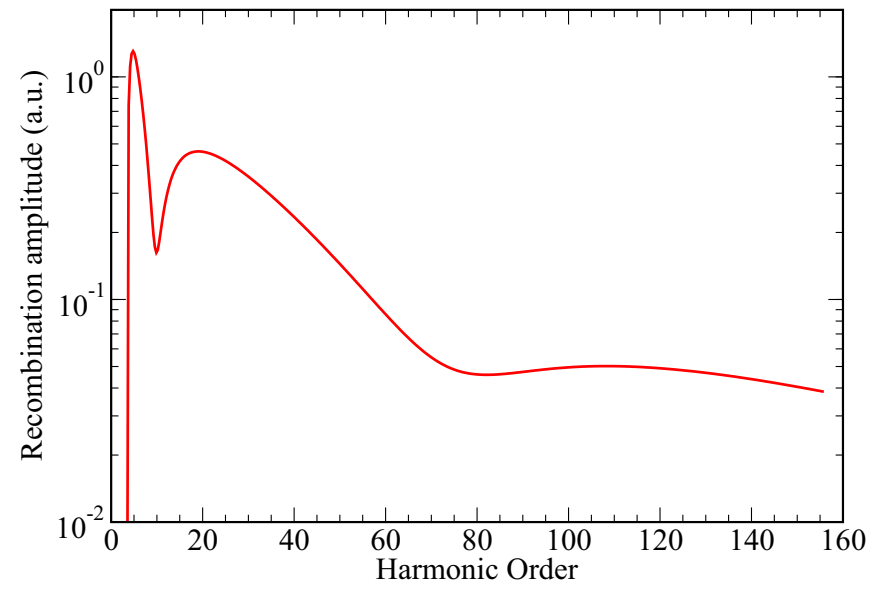

FIG. 7. Dipole recombination matrix element for a continuum electron recombining back to the LUMO+1 autoionizing state. The velocity form of the recombination element is calculated as described in Eq. (13). The energy of the free electron and the ionization potential of the Kohn-Sham state are related to a given harmonic order $n$ using the classical formula $n \omega_{L}=k^{2} / 2+I_{p}$, where $\omega_{L}$ is the frequency of our IR field and $I_{p}$ is the ionization potential of the LUMO+1.

pulses, but with the harmonics due to bound-bound transitions increasing in both sharpness and intensity. At very long pumpprobe delays, the enhancement of the plateau decreases.

\section{High-order harmonic generation: Position of the inner plateau cutoff}

Finally, we return to the question of the position of the cutoff for the excited-state plateau: In essence, there appears to be a suppression of the cutoff harmonics. Minima in harmonic spectra are a well-studied feature in HHG. A number of potential mechanisms could give rise to minima including multichannel [43-46] or structural interference [2,18,19,47-52] effects. Structural interferences can be studied by calculating recombination matrix elements [52-54]. The recombination matrix element of an ionized electron to a bound state of the molecule can be written as

$$
\boldsymbol{d}_{\mathrm{rec}}(\boldsymbol{k})=\langle\chi(\boldsymbol{k}, \boldsymbol{r})|-i \nabla| \Psi(\boldsymbol{r})\rangle,
$$

in the velocity form where $\chi(\boldsymbol{k}, \boldsymbol{r})$ is a plane wave describing the free electron having momentum $\boldsymbol{k}$ and $\Psi(\boldsymbol{r})$ is the wave function of the bound state. We can calculate the absolute value of this amplitude in a given direction $\boldsymbol{e}$ due to a recombination back to any of the field-free Kohn-Sham states of the molecule: In this work we take $\boldsymbol{e}$ to be along the laser polarization direction. Integrating this over all angular variables for $\boldsymbol{k}$ and relating the energy of the free electron and the ionization potential of the Kohn-Sham state to a given harmonic order $n$ using the classical formula gives $n \omega_{L}=k^{2} / 2+I_{p}$, where $\omega_{L}$ is the frequency of our IR field and $I_{p}$ is the ionization potential of the state $\Psi(\boldsymbol{r})$. We plot this recombination amplitude for the LUMO+1 in Fig. 7. Immediately we see that there is a minimum in the recombination amplitude at harmonic 81 , close to the predicted cutoff for recombination to the LUMO+1 for the chosen laser parameters. Suppose we consider the same interaction, but now with the intensity of the IR pulse lowered to $I=6.5 \times 10^{13} \mathrm{~W} / \mathrm{cm}^{2}$. In that case the classical

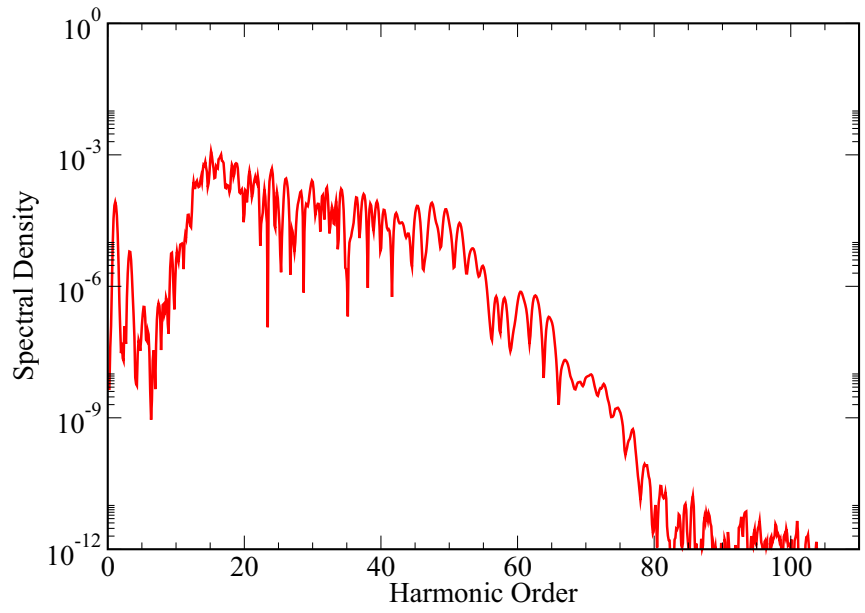

FIG. 8. High-order harmonic generation in acetylene after interaction with a VUV pump pulse and IR probe pulse. The pump pulse is an eight-cycle linearly polarized VUV laser pulse having a wavelength of $\lambda=102 \mathrm{~nm}$ and a peak intensity of $I=1.0 \times 10^{12} \mathrm{~W} / \mathrm{cm}^{2}$. The probe pulse is a five-cycle, linearly polarized IR laser pulse having a wavelength of $\lambda=1450 \mathrm{~nm}$ and a peak intensity of $I=$ $6.5 \times 10^{13} \mathrm{~W} / \mathrm{cm}^{2}$.

cutoff should be at harmonic 53, away from the minimum in the the recombination amplitude to the LUMO+1. The harmonic spectra for this case is shown in Fig. 8 and we see that the observed cutoff agrees with the predicted value. Thus our results support the idea that the main features of this secondary plateau arise due to ionization from and subsequent recombination to the LUMO+1.

\section{CONCLUSION}

We have studied HHG in aligned acetylene molecules using mid-IR laser pulses and shown that an autoionizing state associated with the $3 \sigma_{g} \rightarrow 3 \sigma_{u}$ excitation plays a crucial role whenever the pulse is aligned along the molecular axis. By exciting the molecule with a VUV pulse tuned to this excitation, the harmonic signal in the plateau is dramatically enhanced. This enhancement is associated with ionization from and recombination back to the excited state. Since resonances and autoionizing states are ubiquitous in many molecules this opens up the possibility of controlling reactions using suitable attosecond pulses $[3,55]$. Additionally, since the HHG enhancement using the VUV plus IR pulses is comparable to the spectral density observed using an IR pulse aligned perpendicular to the molecule and since the pump pulse does not appear to have an effect on the response in the perpendicular alignment, the use of the VUV pump should lead to a boost in the overall harmonic signal when considering unaligned samples of molecules.

\section{ACKNOWLEDGMENTS}

This work used the ARCHER UK National Supercomputing Service (http://www.archer.ac.uk) and has been supported by COST Action No. CM1204 (XLIC). P.M. acknowledges financial support through a Ph.D. studentship funded by the UK Engineering and Physical Sciences Research Council. 
[1] J. P. Marangos, J. Phys. B 49, 132001 (2016).

[2] J. Itatani, J. Levesque, D. Zeidler, H. Niikura, H. Pépin, J. C. Kieffer, P. B. Corkum, and D. M. Villeneuve, Nature (London) 432, 867 (2004).

[3] F. Krausz and M. Ivanov, Rev. Mod. Phys. 81, 163 (2009).

[4] P. B. Corkum, Phys. Rev. Lett. 71, 1994 (1993).

[5] K. C. Kulander, K. J. Schafer, and J. L. Krause, in Super-Intense Laser-Atom Physics, edited by B. Piraux, A. L'Huillier, and K. Rzazewski (Plenum, New York, 1993), p. 95.

[6] J. B. Watson, A. Sanpera, X. Chen, and K. Burnett, Phys. Rev. A 53, R1962 (1996).

[7] V. Strelkov, Phys. Rev. Lett. 104, 123901 (2010).

[8] X.-B. Bian and A. D. Bandrauk, Phys. Rev. Lett. 105, 093903 (2010).

[9] S. Beaulieu, S. Camp, D. Descamps, A. Comby, V. Wanie, S. Petit, F. Légaré, K. J. Schafer, M. B. Gaarde, F. Catoire, and Y. Mairesse, Phys. Rev. Lett. 117, 203001 (2016).

[10] R. A. Ganeev, M. Suzuki, M. Baba, H. Kuroda, and T. Ozaki, Opt. Lett. 31, 1699 (2006).

[11] J. Rothhardt, S. Hädrich, S. Demmler, M. Krebs, S. Fritzsche, J. Limpert, and A. Tünnermann, Phys. Rev. Lett. 112, 233002 (2014).

[12] K. Ishikawa, Phys. Rev. Lett. 91, 043002 (2003).

[13] E. J. Takahashi, T. Kanai, K. L. Ishikawa, Y. Nabekawa, and K. Midorikawa, Phys. Rev. Lett. 99, 053904 (2007).

[14] C. Vozzi, R. Torres, M. Negro, L. Brugnera, T. Siegel, C. Altucci, R. Velotta, F. Frassetto, L. Poletto, P. Villoresi, S. De Silvestri, S. Stagira, and J. P. Marangos, Appl. Phys. Lett. 97, 241103 (2010).

[15] R. Torres, T. Siegel, L. Brugnera, I. Procino, J. G. Underwood, C. Altucci, R. Velotta, E. Springate, C. Froud, I. C. E. Turcu, M. Yu. Ivanov, O. Smirnova, and J. P. Marangos, Opt. Express 18, 3174 (2010).

[16] C. Vozzi, M. Negro, and S. Stagira, J. Mod. Opt. 59, 1283 (2012).

[17] M. Negro, M. Devetta, D. Faccialá, S. De Silvestri, C. Vozzi, and S. Stagira, Faraday Discuss. 171, 133 (2014).

[18] M. Lein, N. Hay, R. Velotta, J. P. Marangos, and P. L. Knight, Phys. Rev. Lett. 88, 183903 (2002).

[19] M. Lein, N. Hay, R. Velotta, J. P. Marangos, and P. L. Knight, Phys. Rev. A 66, 023805 (2002).

[20] J. E. Collin and J. Delwiche, Can. J. Chem. 45, 1883 (1967).

[21] P. W. Langhoff, B. V. McKoy, R. Unwin, and A. M. Bradshaw, Chem. Phys. Lett. 83, 270 (1981).

[22] S. Zamith, V. Blanchet, B. Girard, J. Andersson, S. L. Sorensen, O. Björneholm, I. Hjelte, D. Gauyacq, J. Norin, J. Mauritsson, and A. L'Huillier, J. Chem. Phys. 119, 3763 (2003).

[23] L. E. Machado, E. P. Leal, G. Csanak, B. V. McKoy, and P. W. Langhoff, J. Electron Spectosc. 25, 1 (1982).

[24] J. C. Han, C. Ye, M. Suto, and L. C. Lee, J. Chem. Phys. 90, 4000 (1989).

[25] K. Mitsuke and H. Hattori, J. Chem. Phys. 102, 5288 (1994).

[26] M. C. Wells and P. R. Lucchese, J. Chem. Phys. 111, 6290 (1999).

[27] T. Yasuike and S. Yabushita, Chem. Phys. Lett. 316, 257 (2000).

[28] G. Fronzoni, M. Stener, and P. Decleva, Chem. Phys. 298, 141 (2004).
[29] E. Runge and E. K. U. Gross, Phys. Rev. Lett. 52, 997 (1984).

[30] D. Dundas, J. Chem. Phys. 136, 194303 (2012).

[31] A. Wardlow and D. Dundas, Phys. Rev. A 93, 023428 (2016).

[32] A. J. Krueger and N. T. Maitra, Phys. Chem. Chem. Phys. 11, 4655 (2009).

[33] J. P. Perdew and Y. Wang, Phys. Rev. B 45, 13244 (1992).

[34] M. E. Casida, C. Jamorski, K. C. Casida, and D. R. Salahub, J. Chem. Phys. 108, 4439 (1998).

[35] C. Legrand, E. Suraud, and P.-G. Reinhard, J. Phys. B 35, 1115 (2002).

[36] N. Troullier and J. L. Martins, Phys. Rev. B 43, 1993 (1991).

[37] L. Kleinman and D. M. Bylander, Phys. Rev. Lett. 48, 1425 (1982).

[38] M. J. T. Oliveira and F. Nogueira, Comput. Phys. Commun. 178, 524 (2008).

[39] K. Burnett, V. C. Reed, J. Cooper, and P. L. Knight, Phys. Rev. A 45, 3347 (1992).

[40] X. Chu and G. C. Groenenboom, Phys. Rev. A 93, 013422 (2016).

[41] P. Duffy, S. A. C. Clark, C. E. Brion, M. E. Casida, D. P. Chong, E. R. Davidson, and C. Maxwell, Chem. Phys. 165, 183 (1992).

[42] B. K. McFarland, J. P. Farrell, P. H. Bucksbaum, and M. Gühr, Science 322, 1232 (2008).

[43] O. Smirnova, S. Patchkovskii, Y. Mairesse, N. Dudovich, D. Villeneuve, P. Corkum, and M.Y. Ivanov, Phys. Rev. Lett. 102, 063601 (2009).

[44] H. J. Wörner, J. B. Bertrand, P. Hockett, P. B. Corkum, and D. M. Villeneuve, Phys. Rev. Lett. 104, 233904 (2010).

[45] S. Haessler, J. Caillat, W. Boutu, C. Giovanetti-Teixeira, T. Ruchon, T. Auguste, Z. Diveki, P. Breger, A. Maquet, B. Carré, R. Taïeb, and P. Salières, Nat. Phys 6, 200 (2010).

[46] X.-Y. Miao and C.-P. Zhang, Phys. Rev. A 89, 033410 (2014).

[47] T. Kanai, S. Minemoto, and H. Sakai, Nature (London) 435, 470 (2005)

[48] C. Vozzi, F. Calegari, E. Benedetti, J.-P. Caumes, G. Sansone, S. Stagira, M. Nisoli, R. Torres, E. Heesel, N. Kajumba, J. P. Marangos, C. Altucci, and R. Velotta, Phys. Rev. Lett. 95, 153902 (2005).

[49] A.-T. Le, X.-M. Tong, and C. D. Lin, Phys. Rev. A 73, 041402 (2006).

[50] R. Torres, N. Kajumba, J. G. Underwood, J. S. Robinson, S. Baker, J. W. G. Tisch, R. de Nalda, W. A. Bryan, R. Velotta, C. Altucci, I. C. E. Turcu, and J. P. Marangos, Phys. Rev. Lett. 98, 203007 (2007).

[51] K. Kato, S. Minemoto, and H. Sakai, Phys. Rev. A 84, 021403(R) (2011).

[52] A. Etches, M. B. Gaarde, and L. B. Madsen, Phys. Rev. A 84, 023418 (2011).

[53] M. F. Ciappina, A. Becker, and A. Jaroń-Becker, Phys. Rev. A 76, 063406 (2007).

[54] X. Xu, Q. Zhang, W. Hong, P. Lan, and P. Lu, Opt. Express 19, 436 (2011).

[55] F. Lépine, M. Y. Ivanov, and M. J. J. Vrakking, Nat. Photon. 8, 195 (2014). 